\section{EFFECTS OF INTERMITTENT AND CONTINUOUS TRAINING ON THE BLOOD PRESSURE OF NIGERIAN ADOLESCENTS AND CHILDREN}

Wahab B Lawal, ${ }^{1}$ Venkatseswarul Kankanala ${ }^{2}$ Department of Physical and Health Education, FCT College of Education, Zuba, Abuja, Nigeria; '2Department of Physical and Health Education, Ahmadu Bello University, Zaria, Nigeria

10.1136/bjsm.2010.078725.94

Several methods of exercise training are used to promote cardiovascular health. It is not clear as to which of the methods of training are more beneficial in promoting cardiovascular health, especially among children and adolescents. The investigation was therefore, conducted to find out and compare the effects of a 6-week intermittent and continuous training on blood pressure of Nigerian male and female children and adolescents. To achieve this purpose, 15 (10 males and 5 females) children were selected at random from staff school of Ahmadu Bello University, Zaria and another 15 (10 males and 5 females) adolescents were selected from Demonstration Secondary School of the same University. These two groups of children and adolescents were assigned at random to intermittent and continuous training groups. All the subjects were of the same age group, had similar height, weight, blood pressure and activity background. The continuous training group underwent jogging and running at $60 \%$ of maximum heart rate for $30 \mathrm{~min}$, three times a week for 6 weeks. Blood pressure and heart rate of all the subjects were recorded before training and after every 2 weeks of training. The result revealed that both intermittent and continuous groups had a significant decline in blood pressure and heart rate of both male and female children and adolescents. However, intermittent group had a greater decline in blood pressure and heart rate of both male and female children and adolescents than their continuous training group counterparts. This reduction was greater in male and female children than in their adolescent counterparts of both training groups. 